\title{
Double box and hexagon conformal Feynman integrals
}

\author{
B. Ananthanarayan, ${ }^{1 *}$ Sumit Banik $\odot,{ }^{1 \dagger}$ Samuel Friot, ${ }^{2,3 \ddagger}$ and Shayan Ghosh $\odot^{4 \S}$ \\ ${ }^{1}$ Centre for High Energy Physics, Indian Institute of Science, Bangalore 560012, Karnataka, India \\ ${ }^{2}$ Université Paris-Saclay, CNRS/IN2P3, IJCLab, 91405 Orsay, France \\ ${ }^{3}$ Univ Lyon, Université Claude Bernard Lyon 1, CNRS/IN2P3, IP2I Lyon, \\ UMR 5822, F-69622 Villeurbanne, France \\ ${ }^{4}$ Helmholtz-Institut für Strahlen- und Kernphysik and Bethe Center for Theoretical Physics, \\ Universität Bonn, D-53115 Bonn, Germany
}

(Received 20 July 2020; accepted 7 October 2020; published 10 November 2020)

\begin{abstract}
The off-shell massless six-point double box and hexagon conformal Feynman integrals with generic propagator powers are expressed in terms of linear combinations of multiple hypergeometric series of the generalized Horn type. These results are derived from 9-fold Mellin-Barnes representations obtained from their dual conformal Feynman parameter representations. The individual terms in the presented expressions satisfy the differential equation that relates the double box in $D$ dimensions to the hexagon in $D+2$ dimensions.
\end{abstract}

DOI: 10.1103/PhysRevD.102.091901

\section{INTRODUCTION}

It was noted in a recent work [1] that there is a close connection between the Mellin-Barnes (MB) computational technique and the constraints coming from a recently discovered Yangian symmetry of conformal Feynman integrals [2,3]. The authors of [1] argue that the MB method can be used to generate Yangian invariants as sums of residues. However, they also point out, for the important cases of the analytically unknown off-shell massless six-point $D$-dimensional double box integral and related hexagon, that there are complications, both in the MB and Yangian approaches, in identifying the latter integrals as particular combinations of these invariants. Thus, the resolution of these remains a challenging and unsolved problem.

To make a breakthrough in this context, we have extended the computational technique of $[4,5]$ in order to deal with $N$-fold MB integrals. This allows us to solve the problem above from the MB side, by extracting series representations of both the double box and hexagon Feynman integrals with generic propagator powers from their ninefold MB representations in a systematic way. The results are given in terms of linear combinations of multiple

\footnotetext{
*anant@iisc.ac.in

†sumitbanik@iisc.ac.in

*samuel.friot@universite-paris-saclay.fr

ghosh@hiskp.uni-bonn.de
}

Published by the American Physical Society under the terms of the Creative Commons Attribution 4.0 International license. Further distribution of this work must maintain attribution to the author(s) and the published article's title, journal citation, and DOI. Funded by SCOAP. hypergeometric series of the generalized Horn type. We emphasize that the derivation of these linear combinations does not require convergence considerations. Regarding the large number of possible series to build the linear combinations, 2530 in the hexagon case and 4834 for the double box, this amounts to a distinct advantage compared to the corresponding situation in the Yangian bootstrap approach of [1] where the convergence properties of the involved series seem to be a necessary external input.

For each of the ninefold MB integrals, one can obtain hundreds of different linear combinations which are analytic continuations of one another converging in different regions of the nine-dimensional space of the cross-ratios that enter the expressions. Obviously the full set of these linear combinations cannot be presented here. We therefore focus on one example of such expressions for both the double box and hexagon, which is explicitly given in the Supplemental Material [6] to this paper. The individual terms in the presented expressions satisfy the differential equation that relates the double box in $D$ dimensions to the hexagon in $D+2$ dimensions. In addition to solving the thus far unsolved problem of evaluating these complicated Feynman integrals, it is likely that such an approach may yield insights for the solution of the corresponding Yangian constraints.
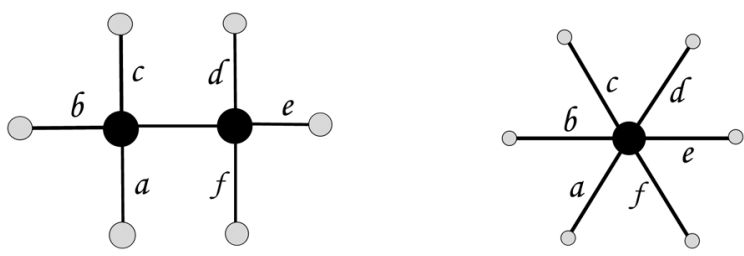

FIG. 1. The double box and hexagon integrals. 


\section{MELLIN-BARNES REPRESENTATIONS OF THE DOUBLE BOX AND HEXAGON}

We follow in our computations the notational conventions of [1] where the six-point off-shell massless double box with generic propagator powers (see Fig. 1 left) is written, in dual momentum space, as

$$
I_{3,3}=\int \frac{\mathrm{d}^{D} x_{0} \mathrm{~d}^{D} x_{0^{\prime}}}{x_{10}^{2 a} x_{20}^{2 b} x_{30}^{2 c} x_{00^{\prime}}^{2 \ell} x_{40^{\prime}}^{2 d} x_{50^{\prime}}^{2 e} x_{60^{\prime}}^{2 f}}=V_{3,3} \phi_{3,3},
$$

with $\phi_{3,3}$ a conformally invariant function of nine cross-ratios $\left(u_{i}, i=1, \ldots, 9\right)$ and $V_{3,3}$ is the prefactor,

$$
V_{3,3}=x_{13}^{2 \ell-D} x_{14}^{D-2 \ell} x_{15}^{-2 d-2 e} x_{16}^{2 d+2 e-2 a} x_{26}^{-2 b} x_{36}^{D-2 c-2 \ell} x_{46}^{2 \ell-2 d-D} x_{56}^{2 d} \text {. }
$$

The dual conformal Feynman parameter representation of the double box is given in [1], where

$$
\phi_{3,3}\left(u_{1}, \ldots, u_{9}, D\right)=Q_{3,3} \int_{0}^{\infty} \mathrm{d} \beta_{2} \mathrm{~d} \beta_{3} \mathrm{~d} \beta_{4} \mathrm{~d} \beta_{5} \frac{\beta_{2}^{b-1} \beta_{3}^{c-1} \beta_{4}^{d-1} \beta_{5}^{e-1}}{X_{2}^{D / 2-\ell} Y^{D / 2-f} Z_{4}^{f}}
$$

with

$$
\begin{gathered}
Q_{3,3}=\frac{\pi^{D} \Gamma(D / 2-\ell) \Gamma(D / 2-f)}{\Gamma(a) \Gamma(b) \Gamma(c) \Gamma(l) \Gamma(d) \Gamma(e)}, \\
X_{2}=\beta_{2} u_{6} u_{9}+\beta_{3} u_{9}+\beta_{2} \beta_{3} u_{1} u_{2} u_{3} u_{9}, \\
Y=u_{8} X_{2}+\beta_{4} u_{9}+\beta_{2} \beta_{4} u_{2} u_{3} u_{9}+\beta_{3} \beta_{4} u_{2} u_{3} u_{4} u_{5} u_{9}+\beta_{5}+\beta_{2} \beta_{5} u_{3}+\beta_{3} \beta_{5} u_{3} u_{5}+\beta_{4} \beta_{5} u_{3} u_{5} u_{7},
\end{gathered}
$$

and

$$
Z_{4}=1+\beta_{2}+\beta_{3}+\beta_{4}+\beta_{5} .
$$

In the expressions above, $x_{i j}^{\mu}=x_{i}^{\mu}-x_{j}^{\mu}$ and the momenta are related to their duals by $p_{j}^{\mu}=x_{j}^{\mu}-x_{j+1}^{\mu}$. Note that the conformal constraints are $a+b+c+\ell=D$ and $d+e+f+\ell=D$.

A similar analysis yields the hexagon integral (see Fig. 1 right) as

$$
I_{6}=\int \frac{\mathrm{d}^{D} x_{0}}{x_{10}^{2 a} x_{20}^{2 b} x_{30}^{2 c} x_{40}^{2 d} x_{50}^{2 e} x_{60}^{2 f}}=V_{6} \phi_{6},
$$

where [1]

$$
\phi_{6}\left(u_{1}, \ldots, u_{9}, D\right)=Q_{6} \int_{0}^{\infty} \mathrm{d} \beta_{2} \mathrm{~d} \beta_{3} \mathrm{~d} \beta_{4} \mathrm{~d} \beta_{5} \frac{\beta_{2}^{b-1} \beta_{3}^{c-1} \beta_{4}^{d-1} \beta_{5}^{e-1}}{Y^{D / 2-f} Z_{4}^{f}}
$$

with

$$
Q_{6}=\frac{\pi^{D / 2} \Gamma(D / 2-f)}{\Gamma(a) \Gamma(b) \Gamma(c) \Gamma(d) \Gamma(e)}
$$

and $V_{6}=x_{15}^{2 f-D} x_{16}^{D-2 a-2 f} x_{26}^{-2 b} x_{36}^{-2 c} x_{46}^{-2 d} x_{56}^{D-2 e-2 f}$.

In this case, the conformal constraint is $a+b+c+d+e+f=D$.

It is straightforward to derive the MB representations of the double box and hexagon from the Feynman parametrizations given in Eqs. (3) and (9) which can be written as

$$
\begin{aligned}
\phi_{3,3}= & \frac{Q_{3,3} u_{8}^{D / 2-\ell}}{\Gamma(D / 2-f) \Gamma(f)} \frac{1}{(2 i \pi)^{9}} \int_{-i \infty}^{+i \infty} \mathrm{d} z_{1} \cdots \int_{-i \infty}^{+i \infty} \mathrm{d} z_{9} \Pi_{i=1}^{9}\left(w_{i}^{z_{i}} \Gamma\left(-z_{i}\right)\right) \Gamma\left(D-f-\ell+z_{1}+\cdots+z_{9}\right) \\
& \times \Gamma\left(b+z_{1}+z_{5}+z_{8}+z_{9}\right) \Gamma\left(c+z_{2}+z_{6}+z_{7}+z_{8}\right) \Gamma\left(d+z_{3}+\cdots+z_{6}\right) \\
& \times \Gamma\left(e+f+\ell-D-z_{4}-\cdots-z_{9}\right) \Gamma\left(-b-c-d-e+D-\ell-z_{1}-z_{2}-z_{3}-z_{5}-z_{6}-z_{8}\right) \\
& \times \frac{\Gamma\left(\ell-D / 2-z_{7}-z_{8}-z_{9}\right)}{\Gamma\left(-z_{7}-z_{8}-z_{9}\right)}
\end{aligned}
$$


and

$$
\begin{aligned}
\phi_{6}= & \frac{Q_{6}}{\Gamma(D / 2-f) \Gamma(f)} \frac{1}{(2 i \pi)^{9}} \int_{-i \infty}^{+i \infty} \mathrm{d} z_{1} \ldots \int_{-i \infty}^{+i \infty} \mathrm{d} z_{9} \Pi_{i=1}^{9}\left(w_{i}^{z_{i}} \Gamma\left(-z_{i}\right)\right) \Gamma\left(D / 2-f+z_{1}+\cdots+z_{9}\right) \\
& \times \Gamma\left(b+z_{1}+z_{5}+z_{8}+z_{9}\right) \Gamma\left(c+z_{2}+z_{6}+z_{7}+z_{8}\right) \Gamma\left(d+z_{3}+\cdots+z_{6}\right) \\
& \times \Gamma\left(e+f-D / 2-z_{4}-\cdots-z_{9}\right) \Gamma\left(-b-c-d-e+D / 2-z_{1}-z_{2}-z_{3}-z_{5}-z_{6}-z_{8}\right),
\end{aligned}
$$

where the $w_{i}$ are combinations of the $u_{i}$ (see Eq. (A20) in [1]). It is implicit here that the integration contours are nonstraight contours which avoid the poles of the gamma functions, in accordance with the commonly used convention [7].

The two integrals in Eqs. (11) and (12) belong to a class of MB integrals for which several series representations of the latter coexist, converging in various regions of the crossratios' nine-dimensional space. Indeed, if one expresses the gamma functions that appear in the integrands of Eqs. (11) and (12) in the form $\Pi_{i} \Gamma\left(A_{i}+\mathbf{a}_{i} \cdot \mathbf{z}\right) / \Pi_{j} \Gamma\left(B_{j}+\mathbf{b}_{j} \cdot \mathbf{z}\right)$ where $\mathbf{z} \doteq\left(z_{1}, \ldots, z_{9}\right)$, it is easy to see that the ninedimensional vector $\boldsymbol{\Delta}=\sum_{i} \mathbf{a}_{i}-\sum_{j} \mathbf{b}_{j}$ is null for each of the MB integrals, which proves the statement above [8,9].

As a final remark, one should note that the conformally invariant functions of the double box in $D$ dimensions and hexagon in $D+2$ dimensions are related by the differential equation

$\partial_{u_{8}} \phi_{3,3}\left(u_{1}, \ldots, u_{9}, D\right)=-\frac{\pi^{D / 2-1}}{\Gamma(\ell)} \phi_{6}\left(u_{1}, \ldots, u_{9}, D+2\right)$

when $D / 2-\ell=1$, which can be easily checked both at the level of the Feynman parameterizations and MB representations above. We will show in the next section that this is also the case, at the level of each term in the linear combinations that form the solutions that we present in this work.

\section{SOLUTION}

We will now extract series representations of both the ninefold MB integrals shown in Eqs. (11) and (12).
This can be done in a systematic way following our new simple and general method that is based on the construction given in $[4,5]$ and which will be presented in detail in [10]. As already mentioned in the Introduction, an important advantage of our method is that the derivation of the linear combinations of multiple hypergeometric series that give the different series representations of $N$-fold MB integrals does not require convergence considerations. In this sense, our method extends, in a geometrical way, the graphical computational approach of $[4,5]$ dedicated to twofold $\mathrm{MB}$ integrals. It is straightforward to derive the possible series representations as well as their total number from our procedure, and therefore to obtain the different analytic continuations, from the series point of view, that can be extracted for a given $N$-fold MB integral. We have checked for this in many cases that are simpler than the double box and hexagon. However, for the ninefold MB integrals under study in the present paper, deriving all the series representations was much too time consuming. Indeed, applying our method to the hexagon, for example, we have obtained hundreds of different series representations built from linear combinations of 26 series that belong to a set of 2530 possible series (note that this total number of possible series has been also obtained from the Yangian analysis of [1]). However, we do know that many more can be derived. It is therefore impossible to list all these possible linear combinations explicitly in one paper and we focus here on the series representations of the double box and hexagon associated to one particular series representation.

For the double box case, the result is

$$
\begin{aligned}
\phi_{3,3}= & \frac{Q_{3,3} u_{8}^{D / 2-\ell}}{\Gamma(D / 2-f) \Gamma(f)}\left(D_{1}+D_{4}+D_{10}+D_{18}+D_{27}+D_{45}+D_{76}+D_{140}+D_{158}+D_{190}\right. \\
& +D_{208}+D_{318}+D_{340}+D_{440}+D_{542}+D_{674}+D_{1063}+D_{1091}+D_{1435}+D_{1581}+D_{1646} \\
& +D_{2382}+D_{3047}+D_{3068}+D_{3786}+D_{4580}+D_{5}+D_{19}+D_{28}+D_{46} \\
& +D_{142}+D_{210}+D_{826}+D_{926}+D_{942}+D_{988}+D_{1094}+D_{1112}+D_{1330}+D_{1449} \\
& \left.+D_{1647}+D_{2436}+D_{3069}+D_{3806}\right),
\end{aligned}
$$

where the $D_{i}$ are explicitly given in Appendix A (see the Supplemental Material [6] ). The index $i$ of the $D_{i}$ corresponds to their ranking in our list of 4834 series that are used to constitute the different series representations of the double box.

The series representation of the hexagon that corresponds to the series shown in Eq. (14) (from the differential equation (13) point of view) is 


$$
\begin{aligned}
\phi_{6}= & \frac{Q_{6}}{\Gamma(D / 2-f) \Gamma(f)}\left(H_{1}+H_{4}+H_{9}+H_{15}+H_{22}+H_{35}+H_{58}+H_{94}+H_{103}+H_{123}\right. \\
& +H_{133}+H_{199}+H_{210}+H_{270}+H_{331}+H_{409}+H_{637}+H_{653} \\
& \left.+H_{838}+H_{925}+H_{960}+H_{1375}+H_{1664}+H_{1675}+H_{2062}+H_{2442}\right) .
\end{aligned}
$$

The $H_{i}$ are also explicitly given in Appendix B (see the Supplemental Material [6] ). As for the $D_{i}$, the index $i$ of the $H_{i}$ corresponds to their ranking in our list of 2530 series that are used to constitute the different series representations of the hexagon.

It is straightforward to see that, taking into account the overall factor, for each of the first 26 individual terms of Eq. (14) that we had reordered to ease this exercise, the differential equation (13) is satisfied when compared to the 26 individual terms of the hexagon shown in Eq. (15). For the 18 remaining terms of the double box, the differential equation is also satisfied because the derivative of the latter with respect to $u_{8}$ gives zero.
Finding the convergence region of the series representation presented in Eq. (14) [resp. (15)] from the intersection of the convergence regions of its 44 series (resp. 26 series) is a very hard problem that we have not solved yet. However, our method also allows to derive one particular series, that we call the master series, whose convergence region we conjecture to be included in both the convergence regions of the series representations given in Eqs. (14) and (15) (the detailed derivation of master series will be discussed in [10]). The latter master series has the following characteristic list [11]:

$$
\begin{aligned}
\left\{n_{1}+n_{2}-n_{3}, n_{3}-n_{2}-n_{5}, n_{3}+n_{4}+n_{5}-n_{1}-n_{6}, n_{6}-n_{3}, n_{1}+n_{2}+n_{6}-n_{3}-n_{4}-n_{7},\right. & \\
& \left.n_{6}-n_{5}-n_{8}, n_{7}+n_{8}-n_{6}, n_{3}-n_{9}, n_{4}+n_{5}+n_{7}+n_{8}-n_{6}-n_{9}, n_{9}, n_{9}-n_{8}, n_{3}, n_{5}, n_{6}, n_{8}, n_{9}\right\},
\end{aligned}
$$

and its arguments are in the order: $\frac{w_{1} w_{6}}{w_{3} w_{8}}, \frac{w_{2} w_{5}}{w_{3} w_{8}}, \frac{w_{3} w_{8}}{w_{5} w_{6}}, \frac{w_{4} w_{8}}{w_{6} w_{9}}, \frac{w_{5} w_{7}}{w_{6} w_{9}}$, $\frac{w_{6} w_{9}}{w_{7} w_{8}}, \frac{w_{8}}{w_{9}}, \frac{w_{7}}{w_{9}}$, and $w_{9}$.

Having obtained the form of the master series, it is now possible to perform numerical checks of the results presented in Eqs. (14) and (15). For this, we do not need to know the convergence region of the master series explicitly, because one can guess particular values of the $w_{i}$ that make it converge. Using these, we have compared the numerical value of both the series representations (14) and (15) with the direct numerical integration of the corresponding Feynman parametrization integrals given in Eqs. (3) and (9), with the help of Mathematica.

The size of the contributions of each series in Eqs. (14) and (15) strongly depends on the values of the powers of the propagators and in some cases one can find dozens of orders of magnitude between two different series, the tiniest contributions being often well under the precision level that can be reached by Mathematica for the numerical integration in a decent time. Therefore, in order to perform relevant numerical checks, we have chosen specific values of the powers of the propagators such that all series of Eqs. (14) and (15) contribute significantly to the total result and we forced the level of accuracy in the numerical integration of the Feynman parametrization integrals such that the error given by Mathematica is smaller, in absolute value, than the least contributing series. This numerically ensures that the contributions of every one of the series of Eqs. (14) and (15) can be tested.
We have done this exercise for different sets of values for both the hexagon and double box, each time obtaining an excellent numerical agreement between the series and the numerical integration, thereby completing the check. Note that this numerical analysis also gives a strong evidence that our conjecture concerning the master series is correct.

\section{CONCLUSIONS}

Series representations of the six-point off-shell massless double box with generic propagator powers, and related hexagon, have been presented in Eqs. (14) and (15). These series representations, which have the form of linear combinations of multiple hypergeometric series of the generalized Horn type, belong to a large set of different series representations that can be systematically derived from our new powerful and simple method of evaluation of multiple MB integrals. All the series representations of this set are analytic continuations of one another. As a check of the results presented in Eqs. (14) and (15), we have shown that each of their individual terms satisfies the differential equation (13). A detailed numerical analysis has been performed to corroborate this check. It is likely that the solutions of the double box and hexagon that we have obtained from our MB analysis may yield insights for solving the corresponding Yangian constraints.

Our method to derive these series representations, that we will describe in a detailed way in [10], is a direct 
extension to the $N$-fold case of the practical computational approach developed in $[4,5]$ for the twofold case. It has the great advantage of selecting the different series that constitute a given linear combination forming a series representation of the $N$-fold MB integral under study without the need of a prior knowledge of the convergence regions of the possible involved series.

Finding the convergence regions of the ninefold hypergeometric series that constitute the series representations presented in this paper is a difficult open problem, but we would like to add that, for each of the linear combinations that our method can produce, one can also derive a master series whose convergence region, we believe, is common to the convergence regions of all the series that constitute the corresponding linear combination [note that the double box and hexagon series representations in Eqs. (14) and (15) have the same master series; see Eq. (16)]. This can dramatically simplify the convergence analysis in the cases where the convergence region of the master series is equal to the convergence region of the series representation and, in the other cases, this considerably simplifies the numerical checks.

\section{ACKNOWLEDGMENTS}

We thank Alankar Dutta and Prateek Sharma for technical assistance. S. G. thanks Collaborative Research Center CRC 110 Symmetries and the Emergence of Structure in QCD for supporting the research through grants.
[1] F. Loebbert, D. Müller, and H. Münkler, Phys. Rev. D 101, 066006 (2020).

[2] D. Chicherin, V. Kazakov, F. Loebbert, D. Müller, and D. 1. Zhong, J. High Energy Phys. 05 (2018) 003.

[3] D. Chicherin, V. Kazakov, F. Loebbert, D. Müller, and D. 1. Zhong, Phys. Rev. D 96, 121901 (2017).

[4] O. Zhdanov and A. Tsikh, Siberian mathematical Journal 39, 245 (1998).

[5] S. Friot and D. Greynat, J. Math. Phys. (N.Y.) 53, 023508 (2012).

[6] See Supplemental Material at http://link.aps.org/ supplemental/10.1103/PhysRevD.102.091901 for the 44 series required for construction of the solution of the double box in Appendix A and for the 26 series required for the construction of the related solution of the hexagon in Appendix B.

[7] V. A. Smirnov, Springer Tracts Mod. Phys. 250, 1 (2012).

[8] O. Zhdanov and A. Tsikh, Dokl. Math. 57, 24 (1998).

[9] M. Passare, A. K. Tsikh, and A. A. Cheshel, Theor. Math. Phys. 109, 1544 (1996).

[10] B. Ananthanarayan, S. Banik, S. Friot, and S. Ghosh (to be published).

[11] H. M. Srivastava and P. W. Karlsson, Multiple Gaussian Hypergeometric Series, Halsted Press (Ellis Horwood Limited, Chichester) (John Wiley and Sons, New York, 1985). 